\title{
Características físicas e químicas dos cachos de cultivares de Vitis rotundifolia ${ }^{1}$
}

\section{Physical and chemical characteristics of grape clusters from Vitis rotundifolia cultivars}

\author{
Sergio Denega²; Luiz Antonio Biasi3 ${ }^{3 *}$; Flávio Zanette ${ }^{3}$; Márcio Furlan Maggi; \\ Sidnei Osmar Jadoski ${ }^{5}$; Sirlei Janine Blaskevicz ${ }^{6}$
}

\begin{abstract}
Resumo
Este trabalho teve como objetivo avaliar as características físicas e químicas dos cachos de oito cultivares de Vitis rotundifolia. As cultivares estudadas foram as seguintes: Topsail, Magnolia, Noble, Roanoke, Magoon, Regale, Bontiful e Dixie. Os cachos utilizados para as análises foram colhidos da coleção de cultivares de Vitis rotundifolia existente na Estação Experimental do Canguiri, da Universidade Federal do Paraná, em Pinhais-PR. Foram avaliadas duas safras, 2004 e 2005, pelas seguintes variáveis: massa total do cacho, de bagas por cacho e do engaço; número de bagas por cacho; massa média das bagas; e diâmetro de baga. O delineamento experimental foi inteiramente ao acaso, com 50 repetições, sendo cada parcela constituída de um cacho. Também foram avaliadas as características químicas do suco: $\mathrm{pH}$, acidez e teor de sólidos solúveis. O delineamento foi inteiramente ao acaso, com 10 repetições, sendo cada parcela constituída pelo suco de cinco cachos. Quanto ao teor de sólidos solúveis totais, a

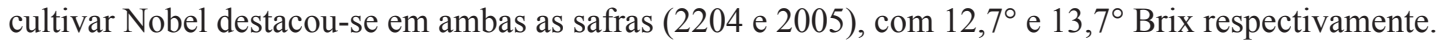
A cultivar Dixie apresentou o maior valor de pH $(3,04)$, em 2004 e a cultivar Magnólia em 2005 foi superior às outras e ao mesmo tempo apresentou a menor acidez nos dois anos. As cultivares Magnólia e Roanoke se destacaram em 2004 e 2005, respectivamente, quanto a massa total do cacho, de bagas e do engaço. Para o número de bagas, em 2004, as cultivares Roanoke e Magnólia apresentaram valores superiores. Já em 2005, as cultivares Roanoke, Regale e Noble foram superiores. A massa média das bagas, em 2004 foi superior nas cultivares Magnólia e Topsail, sendo que esta última cultivar também se destacou em 2005. A cultivar com menor diâmetro das bagas foi a Noble. Conclui-se que as cultivares de Vitis rotundifolia por apresentarem baixo teor de sólidos solúveis, elevada acidez e baixo pH, não são recomendadas para consumo in natura, mas apresentam potencial para o processamento de sucos e geléias. Essas cultivares também apresentam cachos pequenos e com poucas bagas, bastante inferiores aos cachos de outras videiras americanas.
\end{abstract}

Palavras-chave: Brix, $\mathrm{pH}$, acidez, videira, pós-colheita

${ }^{1}$ Parte da Tese de Doutorado do primeiro autor apresentada ao Programa de Pós-Graduação em Agronomia - Produção Vegetal da Universidade Federal do Paraná. Curitiba/PR.

${ }^{2}$ Eng. Agr., Dr. Departamento de Agronomia, Universidade Estadual do Centro Oeste - UNICENTRO. Rua Simeão Camargo Varela de Sá, n 03. Bairro Cascavel, CEP 85.065-090. Guarapuava/PR. E-mail: sdenega@unicentro.br

${ }^{3}$ Eng. Agr., Dr. Professor. Departamento de Fitotecnia e Fitossanitarismo. Universidade Federal do Paraná.- UFPR. Caixa Postal 19061. CEP 81.531-990. Curitiba - PR. Bolsista de Produtividade em Pesquisa do CNPq. E-mail: biasi@ufpr.br

${ }^{4}$ Eng. Agrícola, Dr. Professor. Departamento de Agronomia, Universidade Estadual do Centro Oeste - UNICENTRO. Guarapuava/ PR.. E-mail: mmaggi@unicentro.br

${ }^{5}$ Eng. Agr., Dr. Professor. Departamento de Agronomia, Universidade Estadual do Centro Oeste - UNICENTRO. Guarapuava/PR. E-mail: sjadoski@unicentro.br

${ }^{6}$ Aluna do Curso de Agronomia da Universidade Federal do Paraná - UFPR. Bolsista de Iniciação Científica do CNPq. E-mail: sblas@bol.com.br

* Autor para correspondência 


\begin{abstract}
The goal of this work was to evaluate physical and chemical cluster characteristics of eight Vitis rotundifolia cultivars: Topsail, Magnolia, Noble, Roanoke, Magoon, Regale, Bontiful and Dixie. The evaluated clusters were collected from a preexistent Vitis rotundifolia orchard located at the Canguiri Experimental Station of the Federal University of Parana, in Pinhais-PR. The following variables were evaluated for the 2004 and 2005 crops: total cluster mass, berry mass per cluster, rachis mass, number of berries per cluster and berry diameter. A completely random design with 50 replications was used and each cluster has been considered as a parcel. The chemical characteristics of the juice were evaluated: $\mathrm{pH}$, acidity and total soluble solids. For these analyses, a completely random design with 10 replications was used with 5 clusters per plot. The cultivar Noble stood up from the rest in terms of total soluble solids content for both crops (2004 and 2005) with $12.7^{\circ}$ and $13.7^{\circ}$ Brix respectively. The cultivar Dixie presented the highest $\mathrm{pH}$ (3.04) in 2004 and the cultivar Magnolia in 2005, which present the lower acid content in both years. The cultivars Magnolia and Roanoke stood up in 2004 and 2005 respectively in total cluster mass, mass of berries and rachis mass. In terms of number of berries per cluster, the cultivars Roanoke and Magnolia presented the highest numbers in 2004 while in 2005 the highest numbers were presented by the Roanoke, Regale and Noble cultivars. In 2004, the average berry mass of the cultivars Magnolia and Topsail was superior to the others being the last cited cultivar also superior in this variable in 2005. The cultivar Noble presented the smallest diameter of berries. It was possible to conclude that Vitis rotundifolia cultivars, due to their low total soluble solids content, high acidity and low $\mathrm{pH}$ are not recommended for fresh consumption but present potential for juice, jam and jelly production. Furthermore, the cultivars presented small clusters with few berries, being considered inferior than clusters from other american grapevines.
\end{abstract}

Key words: Brix, PH, acidity, grapevines, post harvest

O gênero Vitis compreende dois subgêneros: Euvitis e Muscadinia. Os subgêneros correspondem a seções de iguais nomes, estando as espécies agrupadas de acordo com a morfologia externa e a origem geográfica. A seção Muscadinia agrupa plantas com 40 cromossomos. As espécies desta seção cujos frutos têm baixo teor de açúcar, são Vitis rotundifolia Michx, a mais importante, Vitis munsoniana Simpson ex Munson e Vitis popenoei Fennell, todas do sudeste dos Estados Unidos e México (GALET, 1993).

As videiras do subgênero Muscadinia são comercialmente importantes para a região sudeste dos Estados Unidos, mas ainda desconhecidas no Brasil. Esta uva possui um sabor e odor característico e o seu interesse comercial consiste no consumo dos frutos in natura, produção de vinhos e sucos (OLIEN, 1990).

A espécie $V$ rotundifolia possui grande resistência à maioria das pragas e doenças da videira, à exceção de uma forma especial de podridão negra. É sensível ao $\mathrm{CaO}$ (óxido de cálcio) e à carência de magnésio, e apresenta difícil hibridação com espécies da seção Euvitis, devido à diferença no número cromossômico. Também apresenta racemos pequenos, bagas médias ou grandes, em geral de cor marrom escuro, às vezes claras e bronzeadas (QUEIROZ-VOLTAN; PIRES, 2003). São pouco açucaradas, com muitos cachos por planta, poucas bagas por cacho, com formação de uma zona de abscisão entre a fruta e a ráquis, casca não estriada e aderente, de lenho duro e maturação escalonada (HIDALGO, 1993; SOUSA, 1996).

Devido à importância da espécie $V$. rotundifolia como uma nova opção para os viticultores de produção orgânica, pela resistência aos principais problemas fitossanitários, o presente trabalho teve como objetivo avaliar as características físicas e químicas dos cachos de diferentes cultivares.

As cultivares estudadas foram Topsail, Magnólia, Noble, Roanoke, Magoon, Regale, Bontiful e Dixie. Os cachos utilizados para as análises foram colhidos da coleção de cultivares de V. rotundifolia, implantada em julho de 1999, no 
Setor de Fruticultura da Estação Experimental do Canguiri, da Universidade Federal do Paraná, em Pinhais-PR.

Foi avaliada a produção das cultivares nas safras de 2004 e 2005 pelas seguintes variáveis: massa total do cacho, de bagas por cacho e do engaço; número de bagas por cacho; massa média e diâmetro de baga. $\mathrm{O}$ delineamento foi inteiramente ao acaso, com 50 repetições, sendo cada parcela constituída de um cacho.

Também foram avaliadas as características químicas do suco pelas seguintes variáveis: $\mathrm{pH}$, acidez e teor de sólidos solúveis. O delineamento foi inteiramente ao acaso, com 10 repetições, sendo cada parcela constituída pelo suco de 5 cachos.

$\mathrm{O} \mathrm{pH}$ foi medido com $\mathrm{pH}$ metro digital de bancada e o teor de sólidos solúveis com refratômetro manual, expresso em graus Brix. A acidez foi determinada pela titulação de $20 \mathrm{~mL}$ do suco mais $80 \mathrm{~mL}$ de água deionizada com $\mathrm{NaOH} 0,1 \mathrm{~N}$ até $\mathrm{pH} 8,1$. O volume de $\mathrm{NaOH}$ gasto na titulação foi transformado para $\mathrm{g}$ de ácido tartárico $\mathrm{L}^{-1}$ pela equação 0,75 x (volume de $\mathrm{NaOH}) / 2$. As avaliações foram realizadas em março de 2004 e abril de 2005.

Os resultados obtidos foram analisados para verificar se atendiam os pressupostos de homocedasticidade (Teste de Bartlett) e de normalidade dos resíduos. Quando atendidos, os dados foram submetidos a análise de variância e as diferenças entre as médias comparadas pelo teste de Tukey a 5\% de probabilidade.

No ano de 2004, observou-se que a cultivar Noble foi superior às demais quanto ao teor de sólidos solúveis totais $\left(12,7^{\circ}\right.$ Brix $)$. Nesta safra, a cultivar Topsail não apresentou produção suficiente para realizar a análise do suco. Em 2005, se destacaram a cv. Topsail $\left(14,2^{\circ}\right.$ Brix $)$ e, novamente a cv. Noble $\left(13,7^{\circ}\right.$ Brix), enquanto que a cv. Regale apresentou os menores teores em ambas as safras (Tabela 1). Os teores de sólidos solúveis observados para quase todas cultivares foram inferiores ao estabelecido pelas normais brasileiras de comercialiazação para o consumo interno, que é de $14^{\circ}$ Brix (GUERRA, 2003). Entretanto, os resultados obtidos foram semelhantes aos já verificados para outras cultivares de videiras de origem americana por Rizzon e Link (2006), que obtiveram $12,9^{\circ}$ Brix para a cultivar Isabel, $12,2^{\circ}$ Brix para a cultivar Bordô e $12,9^{\circ}$ Brix para a cultivar Concord. Valores superiores no teor de sólidos solúveis das cultivares Dixie, Bontiful, Regale e Roanoke foram encontrados no ano de 2007 (SACHI; BIASI, 2008), demonstrando que além do efeito do genótipo, também ocorrem variações entre os anos.

Tabela 1. Teor de sólidos solúveis totais, pH, acidez, número de bagas por cacho e massa média das bagas de oito cultivares de Vitis rotundifolia avaliadas nos anos de 2004 e 2005. Pinhais, PR.

\begin{tabular}{|c|c|c|c|c|c|c|c|c|c|c|}
\hline \multirow[t]{2}{*}{ Cultivar } & \multicolumn{2}{|c|}{ Brix } & \multicolumn{2}{|c|}{$\mathrm{pH}$} & \multicolumn{2}{|c|}{$\begin{array}{l}\text { Acidez (g ác. } \\
\text { tartárico L L-1) }\end{array}$} & \multicolumn{2}{|c|}{$\begin{array}{l}\text { Número de bagas } \\
\text { por cacho }{ }^{1}\end{array}$} & \multicolumn{2}{|c|}{$\begin{array}{c}\text { Massa média das } \\
\text { bagas }(\mathrm{g})\end{array}$} \\
\hline & 2004 & 2005 & 2004 & 2005 & 2004 & 2005 & 2004 & 2005 & 2004 & 2005 \\
\hline Topsail & -- & $14,2 \mathrm{a}$ & -- & $2,78 \mathrm{bc}$ & -- & $9,9 a$ & $3,1 \mathrm{e}$ & $3,6 \mathrm{c}$ & $4,7 \mathrm{a}$ & $4,9 \mathrm{a}$ \\
\hline Magnólia & $11,2 \mathrm{~b}$ & $13,4 \mathrm{bc}$ & $2,86 \mathrm{bc}$ & $3,18 \mathrm{a}$ & $7,8 \mathrm{~d}$ & $4,3 \mathrm{c}$ & $10,3 \mathrm{ab}$ & $3,9 \mathrm{c}$ & $4,9 \mathrm{a}$ & $4,3 \mathrm{~b}$ \\
\hline Roanoke & $9,3 \mathrm{de}$ & $9,9 \mathrm{e}$ & $2,88 \mathrm{bc}$ & $2,73 \mathrm{~cd}$ & $12,7 \mathrm{ab}$ & $10,9 a$ & $10,9 \mathrm{a}$ & $7,6 a$ & $3,6 \mathrm{bc}$ & $4,4 b$ \\
\hline Magoon & $10,7 \mathrm{bc}$ & $10,1 \mathrm{e}$ & $2,71 \mathrm{~d}$ & $2,67 d$ & $13,5 \mathrm{a}$ & $11,0 \mathrm{a}$ & $7,8 \mathrm{c}$ & $5,5 b$ & $3,7 \mathrm{bc}$ & $3,8 \mathrm{~d}$ \\
\hline Regale & $9,2 \mathrm{e}$ & $8,9 \mathrm{f}$ & $2,81 \mathrm{~cd}$ & $2,68 d$ & $11,3 \mathrm{bc}$ & $11,5 \mathrm{a}$ & $5,8 \mathrm{~d}$ & $6,9 a$ & $3,8 \mathrm{~b}$ & $4,0 \mathrm{~cd}$ \\
\hline Dixie & $10,1 \mathrm{~cd}$ & $12,0 \mathrm{~d}$ & $3,04 \mathrm{a}$ & $2,75 c$ & $6,9 \mathrm{~d}$ & $10,4 \mathrm{a}$ & $8,3 \mathrm{bc}$ & $5,9 \mathrm{~b}$ & $3,7 \mathrm{bc}$ & $4,2 \mathrm{bc}$ \\
\hline Noble & $12,7 \mathrm{a}$ & $13,7 \mathrm{ab}$ & $2,95 \mathrm{ab}$ & $2,83 b$ & $9,9 \mathrm{c}$ & $7,0 \mathrm{~b}$ & $5,9 d$ & $6,7 \mathrm{a}$ & $2,4 d$ & $2,2 \mathrm{f}$ \\
\hline Bontiful & 10,1 cde & $12,7 \mathrm{c}$ & $2,52 \mathrm{e}$ & $2,83 b$ & $13,7 \mathrm{a}$ & $7,1 \mathrm{~b}$ & $7,8 \mathrm{c}$ & $4,1 \mathrm{c}$ & $2,9 \mathrm{~cd}$ & $3,3 \mathrm{e}$ \\
\hline CV (\%) & 6,5 & 4,3 & 3,2 & 1,8 & 12,6 & 19,8 & 15,8 & 10,5 & 16,8 & 11,9 \\
\hline
\end{tabular}

Médias seguidas pela mesma letra na coluna não diferem significativamente pelo teste de Tukey a 5\% de probabilidade.

${ }^{1}$ Os dados originais foram transformados em raiz quadrada de $\mathrm{x}+1,0$ para análise. 
Para o pH, no ano de 2004, a cultivar Dixie apresentou o maior valor $(3,04)$, enquanto o menor foi na cultivar Bontiful $(2,5)$, comportamento também observado para as mesmas cultivares em 2007 por Sachi e Biasi (2008). Na safra 2005, a cultivar Magnólia $(3,18)$ destacou-se das demais, sendo que as cultivares Roanoke (2,73), Regale $(2,68)$ e Magoon $(2,67)$ foram estatisticamente semelhantes, com os menores valores de $\mathrm{pH}$ (Tabela 1). Os valores acima foram inferiores aos encontrados por Sato (2007), que obteve $\mathrm{pH} 3,5$ para a cultivar Isabel e 3,7 para a Rubea.

A cultivar Magnólia apresentou a menor acidez nos dois anos, de 7,8 e 4,3 g de ácido tartárico $\mathrm{L}^{-1}$ em 2004 e 2005, respectivamente, enquanto que as cultivares Bontiful, Magoon e Roanoke apresentaram-se mais ácidas, com teores entre 13,7 e $12,7 \mathrm{~g}$ de ácido tartárico $\mathrm{L}^{-1}$, não diferindo entre si, no ano de 2004. No ano de 2005, as cultivares Regale, Magoon, Roanoke e Dixie (10,4 a 11,5 g de ácido tartárico $\mathrm{L}^{-1}$ ) foram as mais ácidas e não diferiram significativamente (Tabela 1). Para uvas destinadas ao consumo in natura, a acidez deve ser entre 6 a $9 \mathrm{~g} \mathrm{~L}^{-1}$, expressa em ácido tartárico (GUERRA, 2003), demonstrando assim a alta acidez da espécie $V$. rotundifolia.

Em relação aos aspectos físicos da uva, observase que, no ano de 2004, a cultivar Magnólia apresentou os maiores valores para a massa total do cacho $(54,4 \mathrm{~g})$ e massa de bagas $(53,5 \mathrm{~g})$ (Tabela 2). No ano de 2005, a cultivar Roanoke foi superior as demais quanto as mesmas variáveis acima. Esses dados caracterizam a espécie $V$. rotundifolia, como de cachos pequenos e com poucas bagas, bastante inferiores aos cachos das cultivares de Vitis vinifera, como 'Tannat' (216,1 g) (RIZZON; MIELE, 2004) e 'Merlot' (161,9 g) (RIZZON; MIELE, 2003), ou ainda da cultivar Isabel (Vitis labrusca) que é uma espécie americana e que apresentou 125,1 g de massa do cacho em um trabalho realizado por Sato (2007).

Tabela 2. Massa total do cacho, massa de bagas por cacho, massa do engaço e diâmetro de baga de oito cultivares de Vitis rotundifolia avaliadas em 2004 e 2005. Pinhais, PR

\begin{tabular}{|c|c|c|c|c|c|c|c|c|}
\hline \multirow[t]{2}{*}{ Cultivar } & \multicolumn{2}{|c|}{$\begin{array}{l}\text { Massa total do cacho } \\
(\mathrm{g})\end{array}$} & \multicolumn{2}{|c|}{$\begin{array}{l}\text { Massa de bagas por } \\
\text { cacho }(\mathrm{g})\end{array}$} & \multicolumn{2}{|c|}{ Massa do engaço ( $g$ ) } & \multicolumn{2}{|c|}{ Diâmetro de baga (mm) } \\
\hline & 2004 & 2005 & 2004 & 2005 & 2004 & 2005 & 2004 & 2005 \\
\hline Topsail & $14,6 \mathrm{e}$ & $17,6 \mathrm{~cd}$ & $14,4 d$ & $17,4 \mathrm{de}$ & $0,2 \mathrm{~d}$ & $0,2 d$ & $18,9 \mathrm{ab}$ & $20,1 \mathrm{a}$ \\
\hline Magnólia & $54,4 \mathrm{a}$ & $17,3 \mathrm{~cd}$ & $53,5 \mathrm{a}$ & $17,0 \mathrm{e}$ & $0,9 \mathrm{a}$ & $0,3 \mathrm{~cd}$ & $19,6 \mathrm{a}$ & $19,3 \mathrm{abc}$ \\
\hline Roanoke & $43,1 b$ & $34,3 \mathrm{a}$ & $42,4 b$ & $33,6 \mathrm{a}$ & $0,7 \mathrm{a}$ & $1,1 \mathrm{a}$ & $17,6 \mathrm{bcd}$ & $19,4 \mathrm{ab}$ \\
\hline Magoon & $30,1 \mathrm{c}$ & $21,3 \mathrm{c}$ & $29,5 \mathrm{c}$ & $21,0 \mathrm{~cd}$ & $0,5 \mathrm{~b}$ & $0,3 \mathrm{bc}$ & $17,7 \mathrm{abcd}$ & $18,0 \mathrm{~cd}$ \\
\hline Regale & $22,4 d$ & $27,9 b$ & $22,3 \mathrm{c}$ & $27,5 b$ & $0,4 \mathrm{bc}$ & $0,4 \mathrm{bc}$ & $18,4 \mathrm{abc}$ & $18,8 \mathrm{bc}$ \\
\hline Dixie & $29,6 \mathrm{~cd}$ & $25,2 b$ & $29,2 \mathrm{c}$ & $24,8 \mathrm{bc}$ & $0,4 \mathrm{~cd}$ & $0,4 \mathrm{bc}$ & 18,1 abcd & $19,0 \mathrm{abc}$ \\
\hline Noble & $13,3 \mathrm{e}$ & $15,6 \mathrm{~d}$ & $13,0 \mathrm{~d}$ & $15,2 \mathrm{e}$ & $0,3 \mathrm{~cd}$ & $0,3 \mathrm{bc}$ & $15,6 \mathrm{e}$ & $15,1 \mathrm{e}$ \\
\hline Bontiful & $22,9 \mathrm{~cd}$ & $14,0 \mathrm{~d}$ & $22,4 \mathrm{c}$ & $13,7 \mathrm{e}$ & $0,5 \mathrm{~b}$ & $0,5 \mathrm{ab}$ & $16,5 \mathrm{cde}$ & $17,4 \mathrm{~d}$ \\
\hline CV (\%) & 42,7 & 29,2 & 42,7 & 29,3 & 55,1 & 18,5 & 7,9 & 5,0 \\
\hline
\end{tabular}

Médias seguidas pela mesma letra na coluna não diferem significativamente pelo teste de Tukey a 5\% de probabilidade. 
No ano de 2004, quanto ao número de bagas por cacho, destacaram-se as cultivares Roanoke $(10,9)$ e Magnólia (10,3), sendo que a cultivar Topsail foi inferior $(3,1)$. Na safra de 2005, produziram mais bagas as cultivares Roanoke $(7,6)$, Regale $(6,9)$ e Noble $(6,7)$ (Tabela 1). Uma das características da espécie $V$. rotundifolia é o baixo número de bagas por cacho (HIDALGO, 1993).

$\mathrm{Na}$ variável massa média das bagas, no ano de 2004, as cultivares Magnólia (4,9 g) e Topsail $(4,7 \mathrm{~g})$ foram superiores, sendo esta última cultivar também superior no ano de 2005 (4,9 g) (Tabela 1). Os valores obtidos para essas cultivares foram superiores aos obtidos por Rizzon et al. (2000) na avaliação da cultivar Isabel (3,0 g).

$\mathrm{Na}$ análise do diâmetro de baga, constatou-se, na safra 2004, que a cultivar Magnólia apresentou valor superior (19,6 mm), destacando-se, na safra 2005, as cultivares Topsail (20,1 mm), Roanoke (19,4 mm), Magnólia (19,3 mm) e Dixie (19,0 mm) não diferindo entre si. Esses valores são superiores aos encontrados por Rizzon et al. (2000) na avaliação da cultivar Isabel (14,8 mm) e por Sato (2007), com as cultivares Isabel e Rubea, de 14,8 e $14,2 \mathrm{~mm}$ respectivamente.

As cultivares de $V$. rotundifolia por apresentarem baixo teor de sólidos solúveis, elevada acidez e baixo pH, não são recomendadas nas condições estudadas para o consumo in natura, mas apresentam potencial para o processamento de sucos e geléias.

As cultivares desta espécie apresentam cachos pequenos e com poucas bagas, bastante inferiores aos cachos de outras videiras americanas.

\section{Referências}

GALET, P. Précis de viticulture. 6. ed. Paris: Imprimerie Dehan, 1993. 582 p.

GUERRA, C. C. Colheita e destino da produção. In: KUHN, G. B. Uva para processamento: produção, aspectos técnicos. Bento Gonçalves: Embrapa Uva e Vinho; Brasília: Embrapa Informação Tecnológica, 2003. p. $123-125$.

HIDALGO, L. Tratado de viticultura general. Madrid: Mundi-Prensa, 1993. 983 p.

OLIEN, W. C. The muscadine grape: botany, viticulture, history and current industry. HortScience, Alexandria, v. 25, n. 7, p. 732-739, 1990.

QUEIROZ-VOLTAN, R. B.; PIRES, E. J. P. A videira. In: POMMER. C. V. Uva: tecnologia de produção, póscolheita, mercado. Porto Alegre: Cinco Continentes, 2003. p. 37-61.

RIZZON, L. A.; LINK. M. Composição do suco de uva caseiro de diferentes cultivares. Ciência Rural, Santa Maria, v. 36, n. 2, p. 689-692, 2006.

RIZZON, L. A.; MIELE. A. Avaliação da cv. Tannat para elaboração de vinho tinto. Ciência e Tecnologia de Alimentos, Campinas, v. 24, n. 2, p. 223-229, 2004.

. Avaliação da cv. Merlot para elaboração de vinho tinto. Ciência e Tecnologia de Alimentos, Campinas, v. 23, p. 156-161, dez. 2003. Suplemento.

RIZZON, L. A.; MIELE, A.; MENEGUZZO, J. Avaliação da uva cv. Isabel para a elaboração de vinho tinto. Ciência e Tecnologia de Alimentos, Campinas, v. 20, n. 1, p. 115121, 2000.

SACHI, A. T.; BIASI, L. A. Maturação dos frutos de quatro cultivares de uvas muscadínias em Pinhais, PR. Scientia Agraria, Curitiba, v. 9, n. 2, p. 255-260, 2008.

SATO, A. J. Comportamento fenológico e produtivo das videiras 'Isabel'e 'Rubea' sobre diferentes portaenxertos no norte do Paraná. Dissertação (Mestrado em Agronomia) - Departamento de Agronomia. Universidade Estadual de Londrina. Londrina, 2007.

SOUSA, J. S. I. de. Uvas para o Brasil. Piracicaba: Fundação de Estudos Agrários Luiz de Queiroz, 1996. $791 \mathrm{p}$. 
